\title{
Features of Management of Innovative Activity of Small Enterprises
}

\author{
${ }^{1}$ Aida A. Safina, ${ }^{2}$ Kristina S. Kuramshina \\ ${ }^{1}$ Kazan Federal University, Institute of Economics and Finance Management \\ ${ }^{2}$ Kazan National Research Technological University, Institute of Innovation Management \\ *Email: mitchl@mail.ru
}

Received: 15th December 2017, Accepted: 20th December 2017, Published: 31st December 2017

\begin{abstract}
This paper deals with the features of innovation planning in small enterprises. In view of the lack of innovative analysis techniques adapted to the conditions and peculiarities of small enterprises, small enterprises neglect to consider innovation activity. Therefore, the paper outlines the basic principles of innovative analysis, including private principles for planning innovation in a small enterprise, as well as elements of a system for a comprehensive analysis of the company's innovation activities. The result of the research is the developed algorithm for using the results of evaluation and analysis of innovation activity in the management of the small enterprise.
\end{abstract}

Keywords: Small Enterprises, Innovation Activity of Small Enterprises, Analysis of Innovation Activity of Small Enterprises, System of Complex Analysis of Innovation Activity of the Enterprise, Principles of Innovation Activity Planning.

\section{Introduction}

A serious obstacle to increasing the innovation activity of small businesses is the lack of innovation management systems and systematic analysis of innovation activities in small businesses.

During the formation of an innovative economy, the leading factors of economic development are scientific and technological progress (STP), the use of innovations, the increase of innovative activity of economic entities. In these conditions, one of the main subjects of innovation is small industrial enterprises that can make a significant contribution to the innovative development of production and the overcoming of crisis phenomena in the economy. The effectiveness of this contribution is related to the ability of small businesses to manage innovation activities, choose effective tools to ensure its continuity, and timely adjust the innovation process. Therefore, there is a need for a systematic analysis of innovation activities. It is an effective tool for enterprises to develop management decisions, identify internal reserves of growth, an instrument for assessing the professionalism and business qualities of managers of small businesses. At the same time, official data and the results of expert assessments indicate that only a little more than $6 \%$ of small enterprises in the manufacturing sector introduce innovations, and the total share of enterprises that carry out innovation activities systematically in the structure of small business is, according to various estimates, from $1.4 \%$ to $3.5 \%$ [1].

Despite the importance of the problem and the considerable attention thereto from the scientific community and practitioners, many problems remain unresolved in this area. So, there are still no innovation analysis methods adapted to the conditions and peculiarities of small enterprises; small enterprises do not consider innovation activity as an independent object of planning, analysis and evaluation; there are no unified approaches to the formation of a set of indicators of analysis and evaluation methods in small enterprises. It should be noted that some areas of analysis that have important theoretical and applied importance, for example, the evaluation of synergistic interaction of elements of innovation activity, have not been developed, and many questions of analysis and evaluation of this sphere change their manifestation in connection with the changed conditions. Therefore, the problem of improving innovation analysis at enterprises remains relevant.

\section{Material and Methods}

Innovation activity is a key factor in ensuring the successful operation of the enterprise.

The following principles should be observed in innovation analysis:

- Data comparability;

- Identity of comparison indicators;

- the use of a unified methodology for calculating indicators and the order of their measurement;

- identity of the time periods taken for data analysis

- adherence to the fundamental rules of analysis.

Using these principles, a comprehensive analysis and assessment of the enterprise's innovation performance indicators based on the study of individual and general results, as well as incomplete information, will help to find the trajectory of innovative development for the enterprise. The planning covers all stages of innovation and relies on both the preliminary and the current and subsequent stages of analysis, which in turn reveals the reality of the plans. Innovation analysis affects not only 
innovation activity, but also describes the influence of innovation processes on the enterprise's economic activity [3]. The system of integrated analysis of innovation activity contains certain elements presented below in Figure 1.

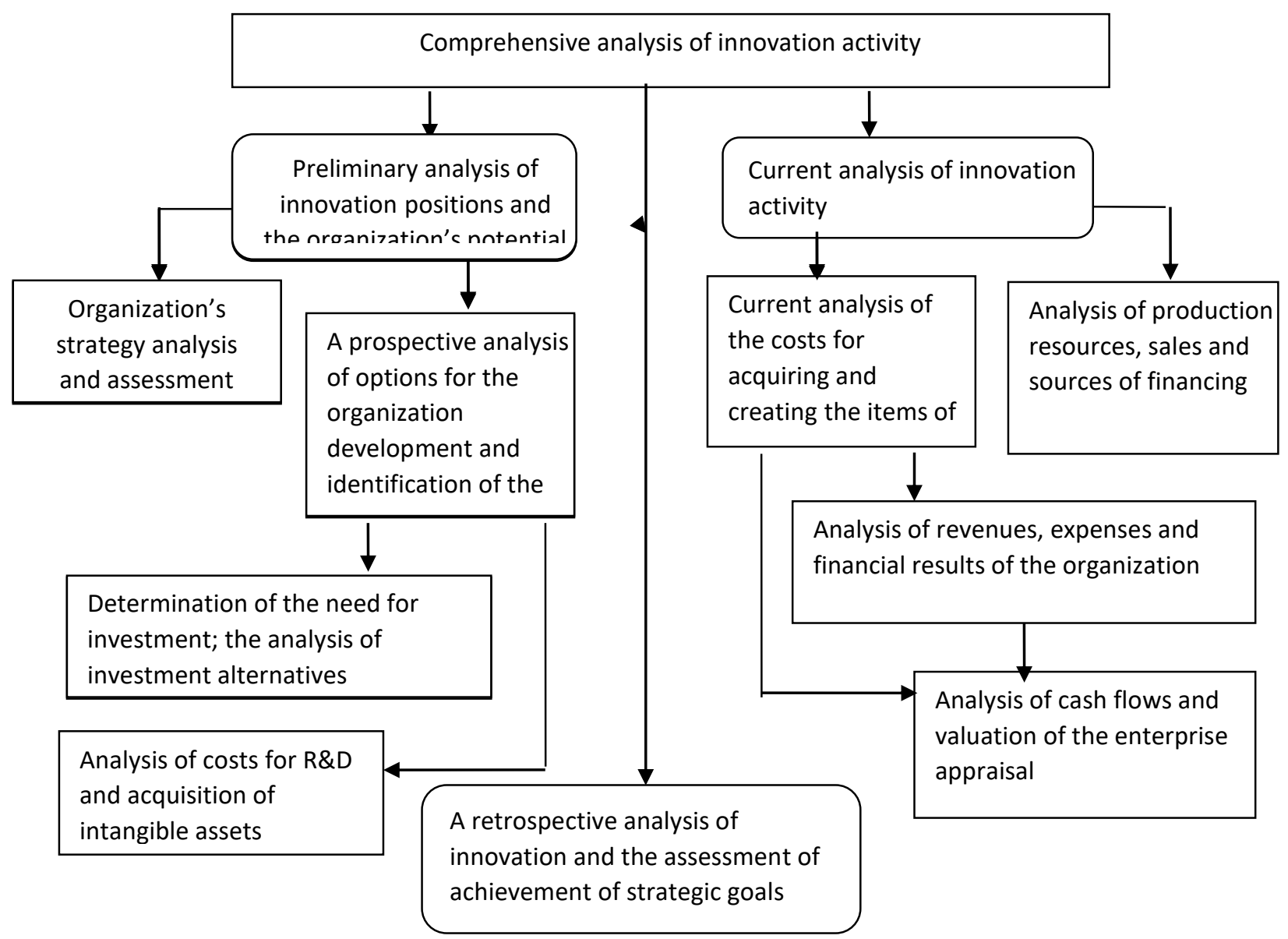

Fig. 1. Elements of the System of the Comprehensive Analysis of Innovative Activity of the Enterprise

It should be noted that a comprehensive analysis of innovation activity is reduced to in-depth consideration of the costs for innovation, income forecasting and their ratio. This analysis is an analytical tool designed to prove or disprove the need for innovative implementation at the current stage of enterprise development, as well as assess the effectiveness and risk of implementation, identify sources of financing for the project, and determine the need to adjust innovation activities in the process of its implementation. One of the methods of innovation analysis is the assessment of the enterprise's innovation potential [4].

As a result of the analysis of existing approaches to the definition of the concept of "innovative potential", it can be concluded that the innovation potential of an enterprise should include such characteristics: innovative potential reflects available resources, their balance and sufficiency for conducting effective innovation activity; innovative potential contains such capabilities of resources, as expected, hidden until a certain time, but which can be activated to implement an innovative strategy; innovative potential includes such components as the organizational system; it also acts as a subsystem of the enterprise's production potential and their development must be balanced; the lag of one of them is a deterrent to innovation; innovative potential is a characteristic of the enterprise's ability to innovation development [5].

\section{Results and Discussion}

The survival of enterprises in the innovation development is related to the ability to manage innovation activity, choose effective tools to ensure its continuity, and timely adjust the innovation process. Currently, management of innovation activities with varying degrees of depth is carried out at each innovative enterprise. In particular, this applies to small businesses, where innovation activity is associated with special innovation risks, which in small business may have slightly different consequences than in large companies. Thus, insignificant risks in comparison with a similar situation in a large company are crucial for the survival of a small enterprise. Currently, as a rule, 
the introduction of innovations in domestic small businesses is not constant but occasional, and their planning is virtually neglected. This is due to a number of reasons, primarily the lack of resources, lack of the necessary tools of strategic management, as well as innovative planning tools adapted to small businesses [6]. It should be noted that innovation activity differs significantly from traditional production and economic activities, especially in small enterprises and must be taken into account in the planning process. There is also a need to highlight innovative planning in the general planning system as a separate subsystem, both at large and medium-sized enterprises, where the scale of innovation is significant, and at small businesses. Indeed, a head of a small enterprise is also interested in reducing the uncertainty and risks that accompany innovation. Small businesses also tend to ensure the concentration of resources in the priority areas of the innovation process, which is achieved through the development of an intra-farm plan. At the same time, its spread caused the emergence of a certain point of view among some economists. It consists in the fact that on-farm planning is replaced by business planning. For example, E.V. Kozlova notes that instead of developing the conceptual foundations of strategic and operational planning, the authors of the publications try to roll over on business planning [7]. As V.S. Novikov noted, innovation planning enters a complex structure of a comprehensive planning, consisting of various plans, in terms of level, goals and content [8]. It is carried out according to the goals (strategic or operational), the subject (production, marketing, finance, personnel), levels (organization, division, program, individual executor), maintenance (technical, economic, food, calendar) and periods (short-, medium-, long-term). Innovative planning at a small enterprise has its own distinctive qualities, which, as a rule, are determined by the size and number of tasks to be solved. Therefore, small businesses in comparison with medium and large enterprises have several other planning needs: they have no right to make mistakes, lack resources and experience, and depend more on external factors. The noted features of innovation planning determine the principles of planning, which establish a set of general rules for the development and effective functioning of the planning system. We will not dwell on general scientific principles of planning, as they are well known. We better define the particular principles that are necessary for the disclosure of the research topic, that is, they form an information field for the analysis and evaluation of innovation in the planning process. Such principles are presented in Figure 2 below.

The considered specific principles are consistently subordinated in a certain way, and interrelated within the innovation activity planning process, which allows structuring the information field and providing information for analysis in the form of a system.

We should agree with I.L. Dimitrov, that the study of the issue related to the determination of features and boundaries of innovative planning is a matter of particular relevance to date [9]. The solution of this issue will allow using more effectively the resources of enterprises in the future to implement their plans and tasks, which can undoubtedly affect both the financial situation and the changing situation on the market, depending on the success of various types of planning.

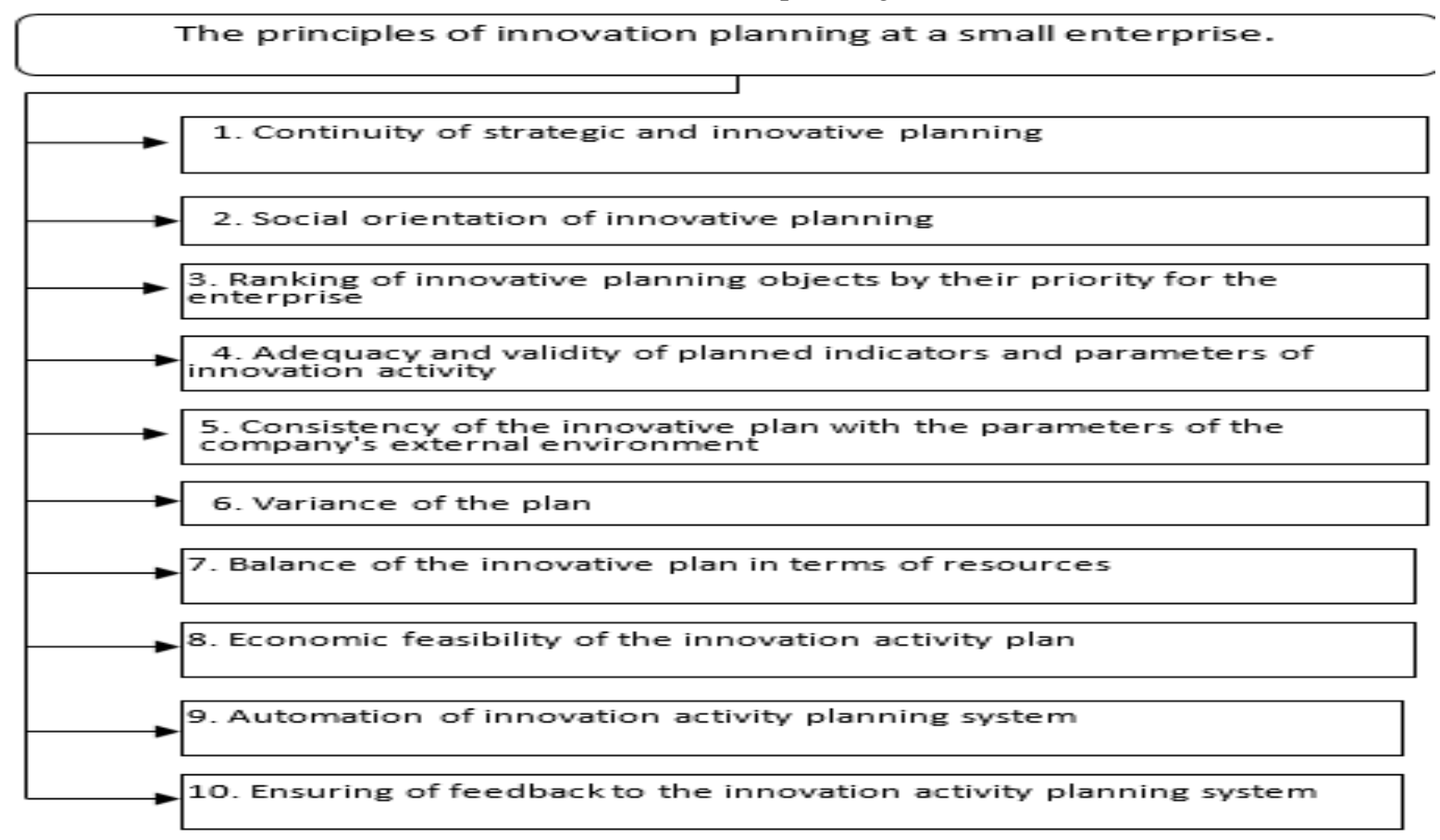


Fig. 2 The Principles of Innovation Planning at a Small Enterprise.

This is especially important for small businesses that have not yet accumulated sufficient experience in this area. Borodushko I., Vasilieva E. note the advantages of planning at small enterprises, which we fully attribute to innovative planning:

- The relative simplicity of organizational and managerial relationships, which predetermines a fairly simple structure of the plan, and the scarcity of managerial staff facilitates the reconciliation of the sections of the plan;

- Mobility and flexibility of the transition to other innovations, ease of change of activities, as well as a narrow specialization in the types of business, as a result of which the plan also does not require differentiation by type of activity;

- Relative ease of reorientation of the type of activity in accordance with the market needs, high possibility of the business adaptation to local conditions [10].

In order to manage and analyze financial and economic and innovative activities in small and medium-sized enterprises, the system "1C: Enterprise 8.0. Production Enterprise Management" is used. It helps to conduct planning, management and accounting of the company's financial, business and innovation activities; as well as planning and coordination of the management of both individual innovative business processes all together, as well as provides automation of staff management, business and tax accounting, and accounting of expenses and incomes for the implementation of innovation activities.

Microsoft Excel usually serves as a universal tool for analytical calculations for innovation activities. This approach does not distinguish the innovation management, including the organizational basis for analysis and evaluation of innovation planning, as a separate subsystem, but disseminates it across different sections of the production and economic plan and the budget of enterprises. Therefore, the analysis of innovation activity gives no complete integrated presentation.

Singling out innovative planning as a separate unit will allow distributing effectively the financial flows to innovative projects in time, training personnel for a set of innovations, using organizational and managerial developments for various innovative projects at the same time, and forecasting and planning additional innovations for the major innovations being implemented [11]. The solution of this problem is the use of an aggregated methodology for analyzing the innovation activity of small and medium-sized enterprises. This synergistic interaction of the components of the innovative system of the enterprise is caused by the presence of distortions, imbalances in the system of the innovative potential of the enterprise and the disruption of the links between the subsystems of the enterprise, which is a deterrent to the innovative development of the enterprise [12].

The emergence of distortions in the innovative system of the enterprise and the disruption of links between the subsystems of the enterprise makes all further investments in the innovation process ineffective, since the innovative system cannot carry out the tasks it faces due to internal imbalances. In this case, there arises the need for a systemsynergetic approach to the analysis of the enterprise's innovative development. This is possible only if synergetic interaction is taken into account in the innovative system of small and medium-sized enterprises. One of the main objectives in the management of innovative activities at the enterprise is the transfer of the innovative system of the enterprise from the unpredictable behavior inherent in dissipative structures to the directed movement determined by attractors (points of attraction).

The most preferred for innovative development is the tandem of the systems of innovation activity of the enterprise and the system of innovation management. Reasonable optimizing managerial decisions are taken in the sphere of innovative activity that do not require significant reorganization, while only the current adjustment of innovation plans and measures in respect to the revealed differences in planning parameters and actual results, identified in the process of analyzing and evaluating innovation activity, are carried out. Figure 3 presents the author's version of the algorithm of using the results of the assessment and analysis of innovation activity in the management of innovation activity of a small enterprise, where $I P_{o}$ is an innovative potential, $E_{t o t}$ is the total effect of innovation activity. 


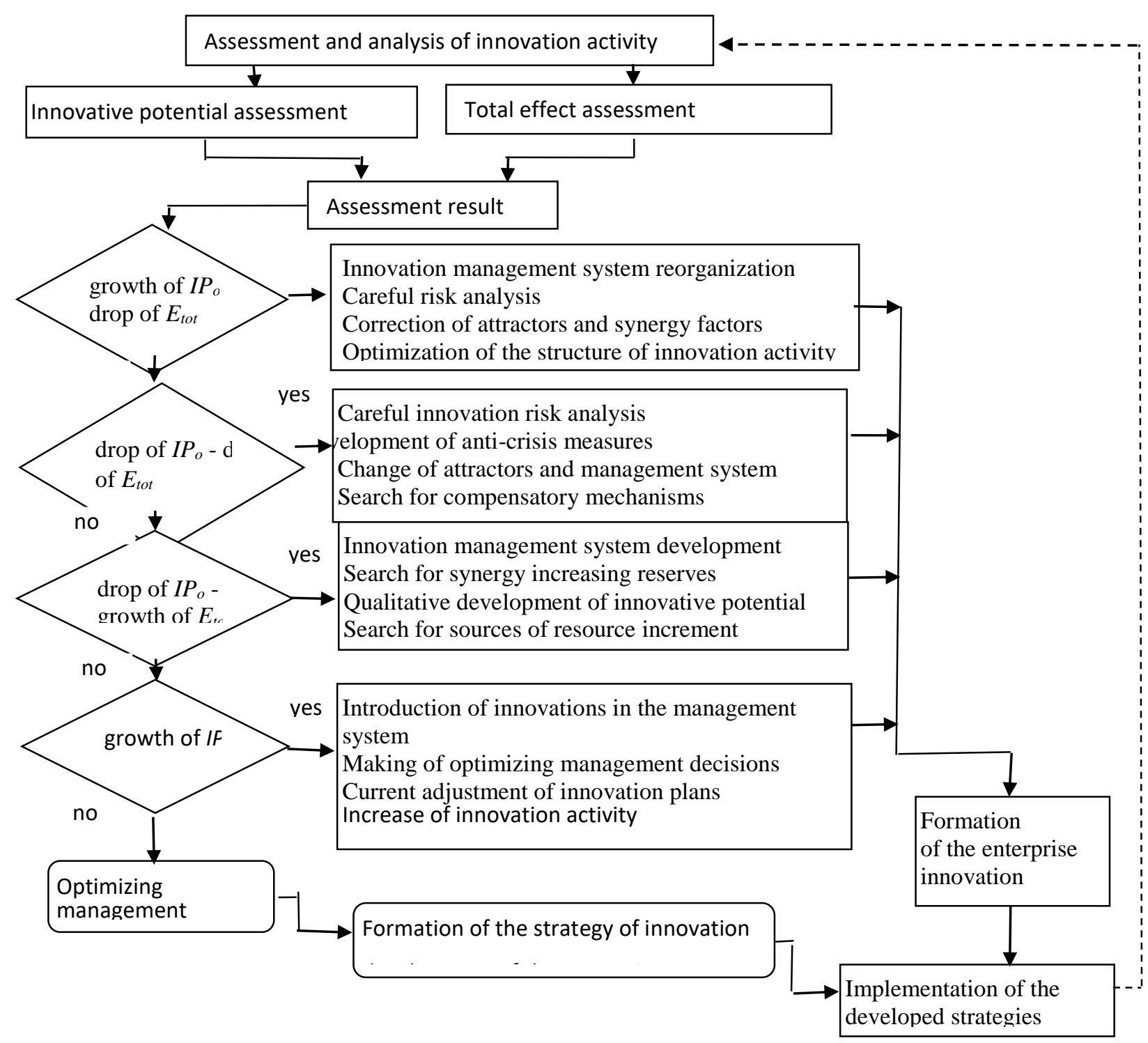

Fig. 3. Algorithm of using the Results of the Assessment and Analysis of Innovation Activity in the Management of a Small Enterprise Innovation Activity

\section{Summary}

Thus, the proposed methodology makes it possible to identify and involve managerial and organizational factors in the development of innovation activities. Their use always growth in the conditions of the economic crisis, since the effect of organizational and managerial innovations (systemic effect) can be quite high, with relatively low costs for their implementation. Therefore, their relevance in the conditions of the modern economy growth, as well as their role in supporting innovation activities with a reduction in investment, due to various external factors, including the economic crisis.

\section{Conclusion}

Investigation of the issue of innovative development of a small and medium-sized enterprise using existing methods of analysis of the financial and economic activities of an enterprise is incomplete, since a qualitative analysis of innovative development is necessary; quantitative indicators, estimated in monetary form, alone do not allow forming a complete picture of innovation activity, innovative development. In this connection, a scheme was proposed for a balanced and harmonious functioning of the innovation activity of a small enterprise and the innovation management system.

\section{Acknowledgements}

The work is performed according to the Russian Government Program of Competitive Growth of Kazan Federal University. 


\section{References}

1. Small innovative business: problems and prospects [Electronic resource] The official website of the program of small business development in St. Petersburg. Access mode: http://smb.gov.ru/measuresupport/programs/celved. 2. Safina A.A., Nikiforova E.G., Shakirova D.S. ESTIMATION PROCEDURE OF INDUSTRIALENGINEERING COMMUNICATION (IEC) / International Business Management. - 2016. volume 10 - issue 23 - p. 5559-5563

3. Kotova T.V., Cherkasova G.G. Innovation analysis: features of information base formation. Bulletin of ASTU. Ser.: Economics, 2010. No.2. pp. 233-236.

4. Vasiukhin O.V., Pavlova E.A. The development of the innovative potential of an industrial enterprise. - M.: Academy of Natural Science, 2010. - p. 176.

5. Ustinov A.E., Nabieva L.G., Davletshina L.M, Safina A.A., Ustinova L. Innovation potential management for economic systems// Academy of Strategic Management Journal, 2016, Volume 15, Special Issue 4.

6. Lebedeva E.E. Features of innovation activity of small businesses / Human. Community. Management. - 2008. No.3. pp. 43-48
7. Kozlova E.V. Strategic and operational planning: theory, methodology and problems of integration into the system http://be5.biz/makroekonomika/index.html [11-2]. 8. Novikov V.S. Innovations in tourism. M.: IC "Akademiia", 2007. - p. 208.

9. Dimitrov I.L. Planning of innovation as the organizing beginning of the innovative project implementation process [Text] / I.L. Dimitrov // Young Scientist. - 2012. - No.12. - pp. 197-198.

10. Borodushko I.V., Vasilieva E.K. // Strategic Planning and Controlling. St. Petersburg "Piter", 2006.

11. Econometric methods for evaluating of open national innovative systems// Authors of DocumentKudryavtseva, S.S., Shinkevich, A.I., Pavlova, A.V., (...), Doronina, F.K., Ishmuradova, I.I // International Journal of Economics and Financial Issues 6 (2), pp. 640-645.

12. Palei, T., Nagimova, A., Zainullina, M., Afonin, P. Estimation of investment requirements in providing potential of reliability of energy provider // Social Sciences and Interdisciplinary Behavior Proceedings of the 4th International Congress on Interdisciplinary Behavior and Social Science, ICIBSOS 2016, Pages 27-30. 